\title{
Structural phase transition and magnetic phase diagram of the lacunar spinel $\mathrm{GaMo}_{4} \mathrm{Se}_{8}$
}

\author{
P. Vir ${ }^{1}$, K. Routledge ${ }^{2}$, N. Cook ${ }^{2}$, P. A. E. Murgatroyd ${ }^{2}$, S. J. Ahmed ${ }^{3}$, S. N. Savvin ${ }^{1}$, J. B. Claridge ${ }^{3}$, and J. Alaria ${ }^{2}$ \\ ${ }^{1}$ Diffraction Group, Institut Laue-Langevin, Grenoble, France, ${ }^{2}$ Department of Physics, University of Liverpool, United Kingdom, \\ ${ }^{3}$ Department of Chemistry, University of Liverpool, United Kingdom
}

$$
\text { vir@ill.fr }
$$

Lacunar spinel is a class of compounds that are derivative of the spinel family, $\mathrm{AB}_{2} \mathrm{X}_{4}$, with some vacancies at the A-site. They are very interesting both crystallographically and with respect to the physical properties as several members exhibit structural phase transition from $F-43 m$ to $R 3 m$ and long-range magnetic ordering at low-temperature.[1,2] Having $R 3 m\left(C_{3 \mathrm{v}}\right.$ symmetry) space group along with long-range magnetism make these compounds interesting in the aspect of spintronics, as they may host Néel-type skyrmions.[3] One such very well-studied compound is $\mathrm{GaV}_{4} \mathrm{~S}_{8}$ that hosts skyrmion with individual size of $22 \mathrm{~nm}$. [3] Here, we report a study on a different member of the lacunar spinel family, $\mathrm{GaMo}_{4} \mathrm{Se}_{8}$ that is expected to have smaller skyrmions size. We performed high-resolution powder neutron diffraction across the structural phase transition $\left(T_{\mathrm{S}}=51 \mathrm{~K}\right)$. Through Rietveld refinement, it is found out that there are two coexisting low-temperature crystal structures with space group $R 3 \mathrm{~m}$ (major phase) and Imm 2 (minor phase) (Fig.1), which is very unique only for $\mathrm{GaMo}_{4} \mathrm{Se}_{8}$.[4] We propose an explanation for the coexisting of both crystal structures through mode-crystallographic and bond-valence sum analysis and postulate that the large strain in the rhombohedral structure is alleviated by the formation of the orthorhombic phase with larger displacive distortion amplitude. Furthermore, we have carried out magnetization measurements and performed magnetic critical behavior analysis. We find that the magnetic transition in $\mathrm{GaMo}_{4} \mathrm{Se}_{8}$ is close to a tricritical mean-field model, and the analysis of the magnetic phase diagram using magneto-entropic map revealed a positive phasefield which might be an indication of the presence of complex magnetic structures such as cycloid or skyrmions states.

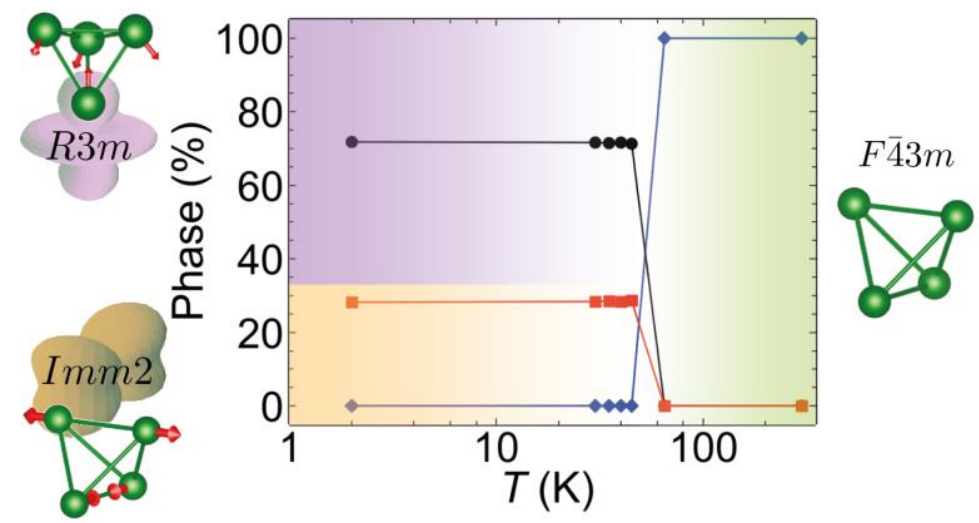

Figure 1. The percentage change in cubic $(F-43 m)$, rhombohedral $(R 3 m)$, and orthorhombic (Imm2) phases of $\mathrm{GaMo}_{4} \mathrm{Se}_{8}$ as a function of temperature. The Mo4 tetrahedron (green color) corresponding to each phase is shown along with the possible distortion direction (red color arrow).

[1] R. Pocha et. al., Chem. Mater. 12, 2882-2887 (2000).

[2] D. I. Khomskii et. al., Chem. Rev. 121, 2992-3030 (2021).

[3] I. Kézsmárki et. al., Nat. Mat. 14, 1116-1122 (2015).

[4] E. C. Schueller et. al., Phys. Rev. Materials 4, 064402 (2020).

Keywords: mode crystallography, phase transition, anisotropic strain 